\title{
Altered renal tubular expression of the complement inhibitor Crry permits complement activation after ischemia/reperfusion
}

\author{
Joshua M. Thurman,1 Danica Ljubanović, ${ }^{2}$ Pamela A. Royer, ${ }^{1}$ Damian M. Kraus, ${ }^{1}$ Hector Molina, ${ }^{3}$ \\ Nicholas P. Barry, ${ }^{1}$ Gregory Proctor, ${ }^{1}$ Moshe Levi, ${ }^{1}$ and V. Michael Holers ${ }^{1}$
}

${ }^{1}$ Department of Medicine, University of Colorado Health Sciences Center, Denver, Colorado, USA. ²Department of Pathology, University Hospital Dubrava, Zagreb, Croatia. ${ }^{3}$ Department of Medicine, Washington University School of Medicine, St. Louis, Missouri, USA.

\begin{abstract}
Ischemia/reperfusion (I/R) of several organs results in complement activation, but the kidney is unique in that activation after I/R occurs only via the alternative pathway. We hypothesized that selective activation of this pathway after renal $I / R$ could occur either because of a loss of complement inhibition or from increased local synthesis of complement factors. We examined the relationship between renal complement activation after $I / R$ and the levels and localization of intrinsic membrane complement inhibitors. We found that loss of polarity of complement receptor 1-related protein y (Crry) in the tubular epithelium preceded activation of the alternative pathway along the basolateral aspect of the tubular cells. Heterozygous gene-targeted mice that expressed lower amounts of Crry were more sensitive to ischemic injury. Furthermore, inhibition of Crry expressed by proximal tubular epithelial cells in vitro resulted in alternative pathway-mediated injury to the cells. Thus, altered expression of a complement inhibitor within the tubular epithelium appears to be a critical factor permitting activation of the alternative pathway of complement after I/R. Increased C3 mRNA and decreased factor $\mathrm{H}$ mRNA were also detected in the outer medulla after $I / R$, suggesting that altered synthesis of these factors might further contribute to complement activation in this location.
\end{abstract}

\section{Introduction}

Complement activation has been shown to be an important event in the development of ischemic acute renal failure (ARF) in mice. Studies in complement-deficient mice have shown that these mice are protected from renal failure after ischemia/reperfusion (I/R) $(1,2)$, and that generation of the anaphylatoxin C5a (3) and the membrane attack complex (2) may contribute to the pathogenesis of ischemic ARF. Treatment with agents that inhibit the complement cascade at specific steps during the activation cascade has proven effective at ameliorating ischemic $\operatorname{ARF}(3,4)$. Determining the initiating mechanisms of complement activation after renal $\mathrm{I} / \mathrm{R}$ is essential to the effective use of complement inhibitors for the treatment and prevention of ischemic ARF.

Complement activation after renal I/R occurs via the alternative pathway (1) and is independent of natural antibody (5). In contrast, I/R injury of other organs such as the heart, intestine, and skeletal muscle appears to involve activation of the classical pathway of complement. Complement activation after intestinal $\mathrm{I} / \mathrm{R}$, for example, is the result of natural antibodies that bind to neoantigens exposed within the ischemic bowel $(6,7)$. Cardiac $\mathrm{I} / \mathrm{R}$ also appears to involve classical pathway activation by antibodies that recognize newly exposed antigens (8). Renal I/R therefore involves unique mechanisms of complement activation.

Nonstandard abbreviations used: ARF, acute renal failure; ATN, acute tubular necrosis; Crry, complement receptor 1-related protein y; DAF, decay-accelerating factor; I/R, ischemia/reperfusion; LDH, lactate dehydrogenase; PTEC, proximal tubular epithelial cell; SUN, serum urea nitrogen.

Conflict of interest: The authors have declared that no conflict of interest exists. Citation for this article: J. Clin. Invest. 116:357-368 (2006). doi:10.1172/JCI24521.
The alternative pathway is ordinarily activated at low levels via an enzymatic process, called "tickover," which results in continuous low-level production of C3b. When C3b binds covalently to an activating surface, such as a bacterial cell wall, it is stabilized such that it can combine with factor B to create the alternative pathway C3 convertase. Such activation of the alternative pathway does not usually cause injury to self cells due to the presence of membranebound and fluid-phase complement regulatory proteins (9). These inhibitors are essential to prevent complement-mediated injury to the host, and deficiency of complement inhibitors can lead to spontaneous injury. For example, humans, pigs, and mice deficient in the fluid-phase factor $\mathrm{H}$ spontaneously develop membranoproliferative glomerulonephritis (10), the expression of dysfunctional CD46 is associated with development of the hemolytic uremic syndrome $(11,12)$, and paroxysmal nocturnal hemoglobinuria is associated with deficiencies of decay-accelerating factor (DAF, also known as CD55) and CD59 (13).

Local synthesis of complement components has also emerged as an important cause of complement activation and tissue injury in some models. For instance, in a murine renal transplant model, synthesis of $\mathrm{C} 3$ by the kidney was found to contribute to complement activation within the tubulointerstitium and, as opposed to serum C3, to be essential to graft loss (14).

Renal I/R in the mouse (1) and in humans (15) leads to the marked deposition of complement activation products along the tubular basement membrane. Although several inhibitors of complement activation are present within the mouse kidney, only complement receptor 1 -related protein y (Crry) is present on mouse tubular epithelial cells (16), the cells primarily injured during I/R. DAF and CD59 expression is limited to the glomeruli and 

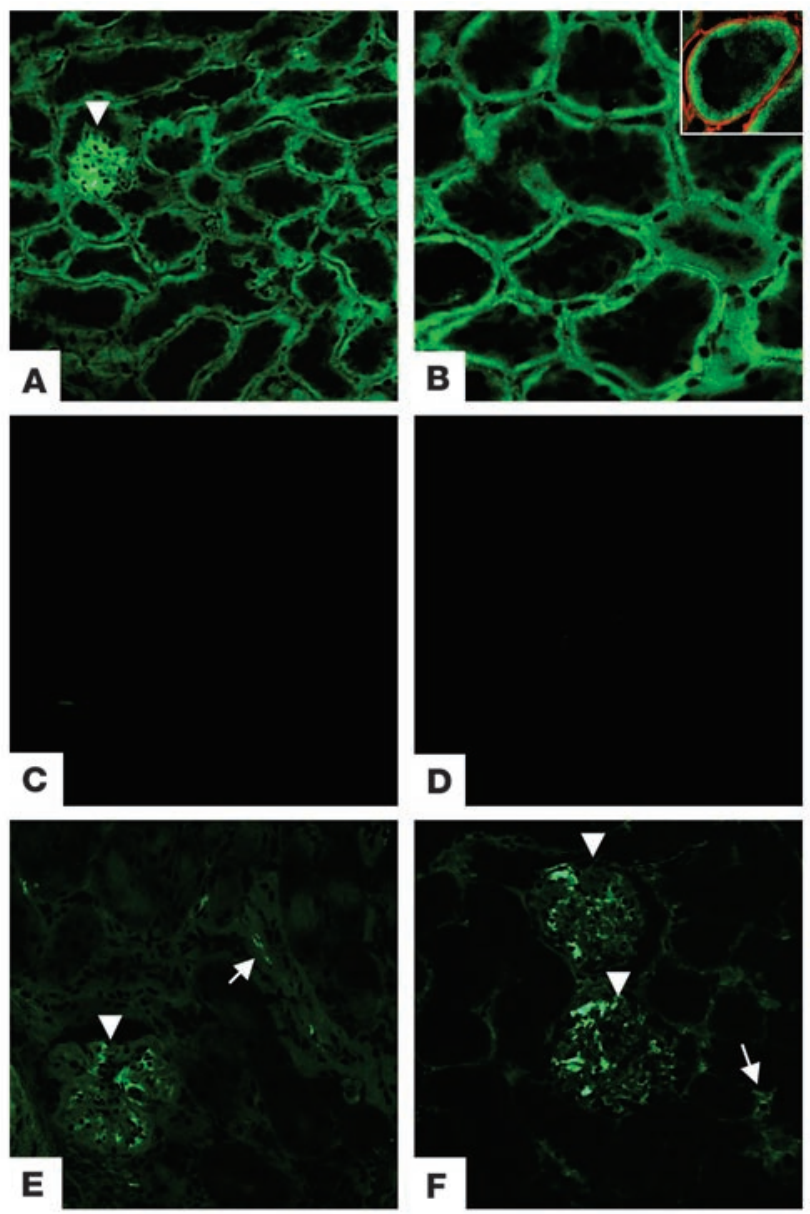

renal arteries $(17,18)$. Deficiency in DAF alone or DAF and CD59 results in vascular complement activation after $\mathrm{I} / \mathrm{R}$ and worse renal injury $(19,20)$. However, substantial vascular complement activation does not occur in wild-type mice after I/R (2). Therefore, in the normal host these particular inhibitors must retain their function, preventing further injury. In contrast, the activation of complement along the tubular basement membrane of wild-type mice after I/R suggests that local inhibition by Crry is either hindered or overwhelmed.

Given the importance of membranebound Crry for the prevention of alternative pathway complement activation and tubular injury, we hypothesized that altered expression or localization of Crry by tubular epithelial cells might contribute to the activation of complement after I/R, or that local synthesis of complement C3 or factor B in response to I/R could overwhelm tubularintrinsic Crry. Therefore, we set out to study the effects of renal ischemia upon Crry and the relation of changes in Crry expression, local synthesis of complement components, and complement activation. We found that altered tubular expression of Crry precedes, and appears to permit, complement activation along the basolateral aspect of

\section{Figure 1}

Localization of membrane-bound complement inhibitors within the mouse kidney. Three different membrane-bound complement inhibitors are located within the mouse kidney. (A) Crry is expressed in the glomeruli (arrowhead) and in tubules. (B) Crry in the renal tubules is polarized to the basolateral aspect of the tubules. Inset: Dual staining for Crry (green) and type IV collagen (red) demonstrates that expression of Crry is adjacent to the tubular basement membrane. Staining with secondary antibody alone in unmanipulated kidneys (C) and kidneys subjected to ischemia and 2 hours of reperfusion (D) demonstrate the specificity of staining with the anti-Crry antibody. (E) CD59 and (F) DAF are also expressed in glomeruli (arrowheads). In addition, these inhibitors may be expressed in the small blood vessels (arrows), but not in tubules. Original magnification, $\times 200$ (A and C-F); $\times 400$ (B).

the tubules. Furthermore, $\mathrm{C} 3$ mRNA was increased and factor $\mathrm{H}$ mRNA was decreased in the outer medulla after $I / R$, indicating that altered synthesis of these factors might further contribute to alternative pathway activation at this location. Understanding the mechanisms whereby intrinsic complement inhibitors are unable to control activation may help in the rational use of complement inhibitors as therapeutics for the prevention of ischemic ARF. These studies may also further our understanding of more general inflammatory responses to injury.

\section{Results}

Localization of intrinsic complement inhibitors within the kidney. Frozen sections of kidneys from unmanipulated mice were examined by immunofluorescence to determine the localization of the membrane-bound complement inhibitors. Consistent with previous reports (14), Crry was heavily expressed within the glomeruli as well as the tubules (Figure 1). Crry in the proximal tubules was polarized to the basolateral surface of the epithelial cells. Expression was contained within the basement membrane, and no Crry was detectable within the cytoplasm or brush border. To further evaluate Crry expression by proximal tubular cells, immunogold labeling and evaluation by electron microscopy were performed on rat kidneys (Figure 2). Labeling within both the apical (34 \pm 4 particles/field, $n=22$ fields) and basal regions (67 \pm 8 particles/ field, $n=19$ fields) was significantly above background labeling with nonimmune serum (12 \pm 2 particles/field, $n=10$ fields; $P<0.01$ versus apical $P<0.001$ versus basal labeling with anti-Crry,
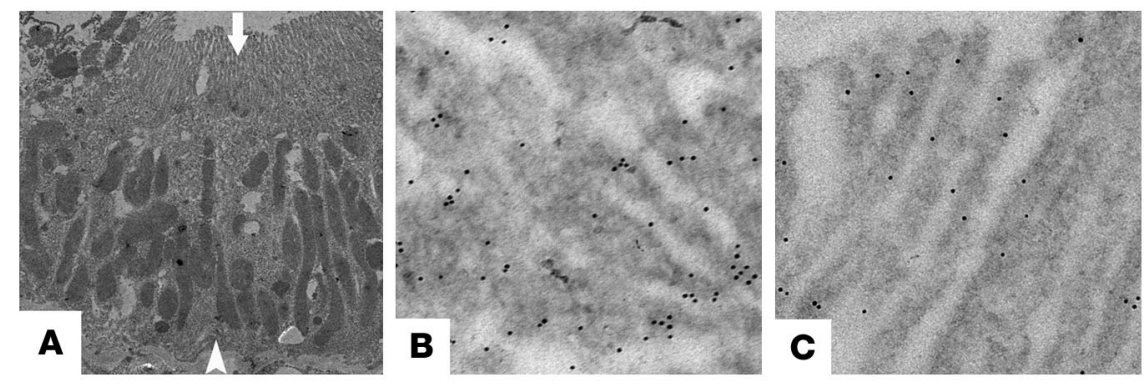

\section{Figure 2}

Immunogold localization of Crry in PTECs. Immunogold labeling of Crry in kidney sections was performed, and the labeling of PTECs (A) was examined (arrow, brush border; arrowhead, basal cell surface). Gold particles could be seen clustered along the basal aspect of the cells (B), and was present to a lesser degree in the apical brush border (C). Original magnification, $\times 1,200(\mathbf{A}), \times 18,500(\mathbf{B}$ and $\mathbf{C})$. 
A

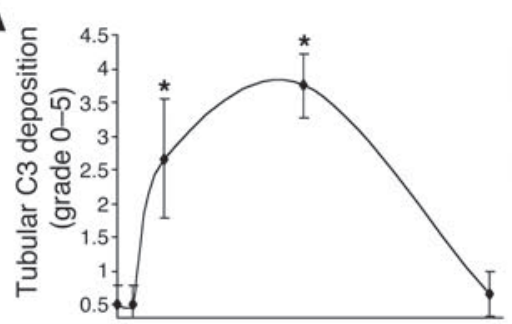

B

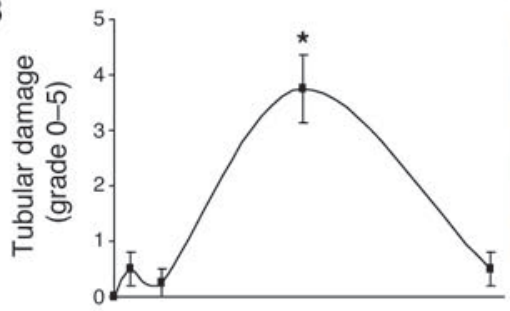

C

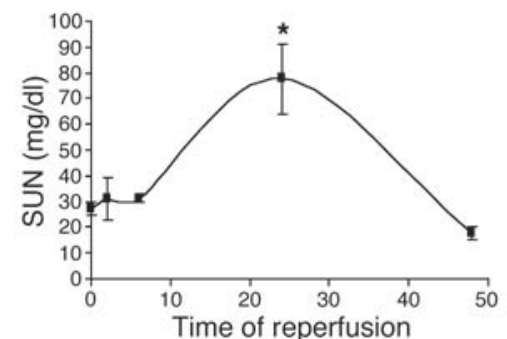

Baseline
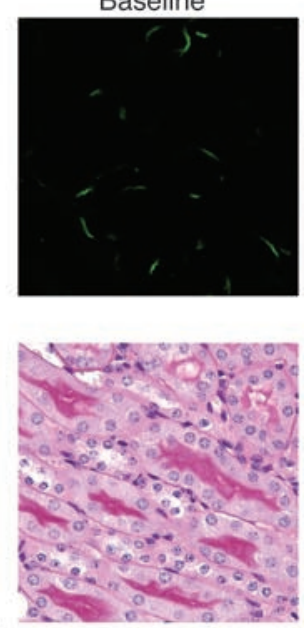

D

Baseline
$\mathrm{Oh}$

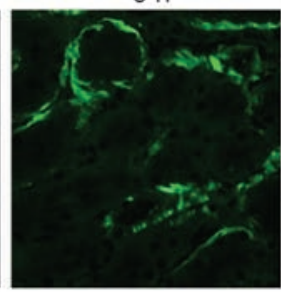

$6 \mathrm{~h}$

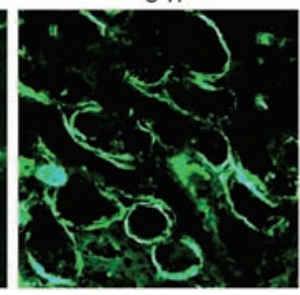

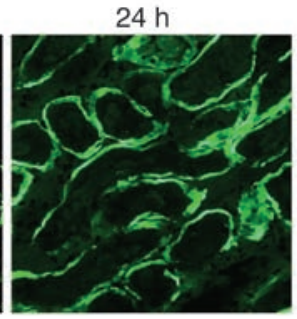
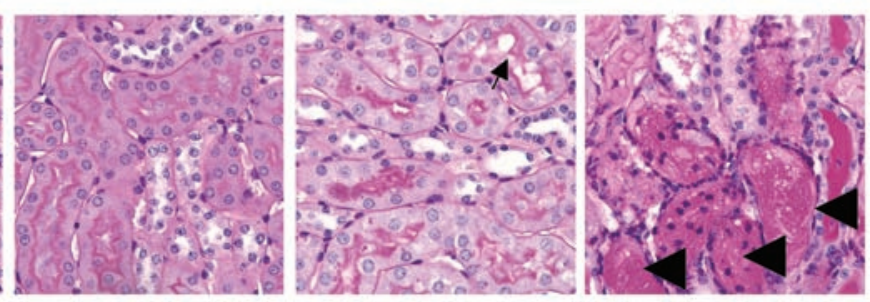
$6 \mathrm{~h}$ $24 \mathrm{~h}$ - 40 kDa C3 fragment

Figure 3

The kinetics of complement activation after I/R. (A) Complement deposition along the tubular basement membrane was assessed by immunofluorescence after ischemia and variable times of reperfusion. Staining was graded by a renal pathologist according to the percentage of tubules in the outer medulla with $\mathrm{C} 3$ deposited along most of the basement membrane. The extent of C3 deposition was significantly increased by 6 hours of reperfusion and peaked at 24 hours. (B) Tubular damage as assessed by light microscopy and (C) SUN peaked at 24 hours of reperfusion and had returned to baseline by 48 hours of reperfusion. Epithelial cell swelling and disruption of the brush border (arrow) were evident by 6 hours, and necrosis (arrowhead) was evident by 24 hours. (D) Western blot analysis of whole-kidney lysates demonstrates that the approximately 40-kD C3 activation fragment increased after 24 hours of reperfusion. ${ }^{*} P<0.05$ versus baseline. $n=4$ for each time point. Original magnification, $\times 200(A), \times 400(B)$.

unpaired Student's $t$ test). Labeling in the region of the basolateral cell surface was significantly higher than that within the apical brush border $(P<0.001$, unpaired Student's $t$ test $)$. As previously reported $(17,18)$, DAF and CD59 were expressed within the glomeruli and vasculature, but not in the tubules (Figure 1).

Complement activation is an early event after renal $I / R$. Mice were subjected to 24 minutes of bilateral renal ischemia or sham surgery and were sacrificed after $0,2,6,24$, and 48 hours of reperfusion. Serum was collected for serum urea nitrogen (SUN) measurements, and kidneys were evaluated for tubular injury and complement C3 deposition. Consistent with previous reports, sham-treated animals demonstrated small areas of $\mathrm{C} 3$ deposition (Figure 3A) along the tubular basement membrane of approximately $10-15 \%$ of the tubules in the cortex and outer stripe of the outer medulla $(1,21)$.

When tubules within the outer medulla were examine by fluorescent microscopy, a significant increase in the percentage of tubules showing complement deposition along the tubular basement membrane was seen by 6 hours of reperfusion (Figure 3A), similar to results previously reported by other groups (22). The degree of tubules showing complement deposition peaked at 24 hours of reperfusion, involving $46-75 \%$ of the tubules, and returned to baseline by 48 hours of reperfusion. Western blot analysis of total kid- ney lysates demonstrated a clear increase in complement activation products after ischemia and 24 hours of reperfusion (Figure 3D).

Although some epithelial cell swelling and brush border injury were evident within 6 hours of reperfusion (Figure 3B), the acute tubular necrosis (ATN) grade did not significantly increase until after 24 hours of reperfusion and returned to baseline by 48 hours of reperfusion (Figure $3 \mathrm{~B}$ ). Thus, complement activation is an early event that precedes severe morphological injury after I/R. Although SUN levels did not significantly increase until after 24 hours of reperfusion (Figure 3C), this was expected because serum markers do not reflect changes in renal function until a new steady state is reached.

Crry levels in the whole kidney do not significantly change after $I / R$. Crry is present in multiple isoforms in the kidney (23). Analysis of protein from immortalized proximal tubular epithelial cells (PTECs) by Western blot revealed that most of the Crry in proximal tubular cells was of the 53-kDa isoform (Figure 4A). Protein from rat kidneys demonstrated that $53-\mathrm{kDa}$ Crry was present in basolateral membrane-enriched fractions but not in brush border-enriched fractions (Figure 4B). Blotting for type IIa Na/Pi cotransporter, which is known to be expressed on the apical brush border, was stronger on the brush border-enriched fraction. As further evidence that it is the $53-\mathrm{kDa}$ isoform that is expressed by PTECs, the 
A

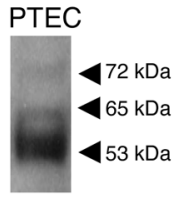

B

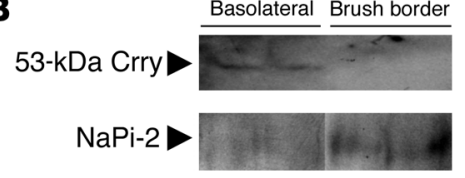

C
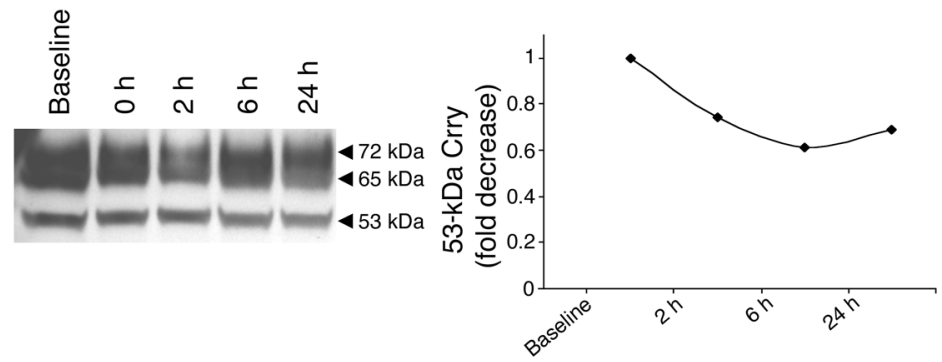

D

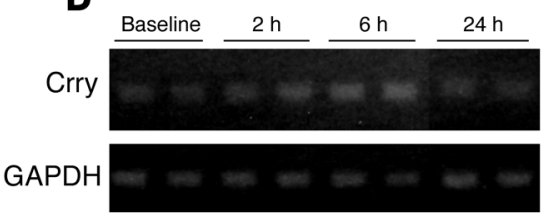

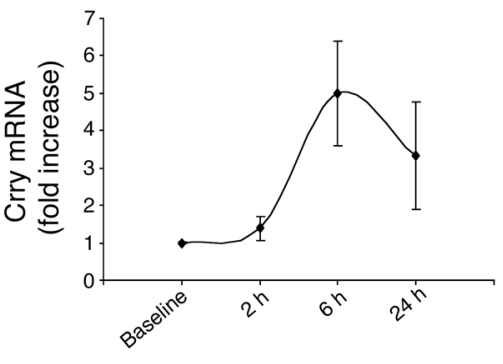

\section{Figure 4}

Crry levels in the kidney after I/R. (A) Lysates from a proximal tubular cell line demonstrate that Crry in the tubular cells is composed almost entirely of the 53-kDa isoform. (B) Protein lysates from rat kidneys enriched for basolateral membrane proteins or brush border membrane proteins demonstrated that $53-\mathrm{kDa}$ Crry resides almost entirely in the basolateral fraction. In contrast, type Ila $\mathrm{Na} / \mathrm{Pi}$ cotransporter (NaPi-2), which is present in the brush border, was more abundant in the brush border-enriched fraction. (C) Total kidney lysates were blotted for Crry after ischemia and variable times of reperfusion. Densitometry of 53-kDa Crry was averaged for duplicate blots and showed a trend toward decreased expression after 6 hours of reperfusion. (D) Semi-quantitative PCR of cDNA made from the outer medulla demonstrated a trend toward increased transcription of Crry after 6 hours of reperfusion. other 2 Crry isoforms were not detected in the basolateral membrane-enriched fraction. When protein was isolated from wholekidney sections, little difference in overall levels of Crry was discerned (Figure 4C), likely due to the fact that Crry is expressed in glomeruli and in tubules throughout the kidney. However, a trend toward decreased expression of the $53-\mathrm{kDa}$ isoform was seen after 6 hours of reperfusion. Semi-quantitative PCR was performed on tissue isolated from the outer medulla (Figure 4D). A trend toward increased mRNA for Crry was seen after 6 hours of reperfusion.

Altered Crry localization precedes complement activation. As discussed above, Crry is the only complement inhibitor expressed on the surface of renal tubules. At baseline, Crry was polarized to the basolateral aspect of the renal tubules (Figure 1), abutting the site of occasional focal $\mathrm{C} 3$ deposition along the tubular basement membrane (Figure 5A). In kidneys subjected to 24 minutes of ischemia this polarity was lost before reperfusion, and Crry could be seen within the tubule lumen (Figure 5B). As shown in Figure 3, the tubules showed signs of injury at these early time points but did not yet show necrosis. The altered Crry expression is therefore a biochemical change of non-necrotic tubules. After 6 and 24 hours of reperfusion (Figure 5, C and D), individual tubules demonstrated little remaining Crry. Those tubules that no longer demonstrated Crry expression had C3 deposited along their circumference. By 48 hours of reperfusion Crry still appeared in the cytosol but was also seen concentrated at the basolateral surface, and complement deposition had nearly returned to baseline (Figure 5E).

Because Crry appeared in the tubular lumen after ischemia, we examined the urine for the presence of Crry. It is known that PTECs shed F-actin-containing membrane vesicles into the tubular lumen after I/R (24). We found that the urinary sediment of mice subjected to I/R contained both F-actin and Crry (Figure 5F). This indicates that loss of Crry in the blebs and vesicles shed in the urine after I/R partially accounts for the absence of Crry in some tubular segments.
Lower-powered views of kidneys stained for Crry and C3 demonstrated that Crry and C3 did not colocalize along the tubules. At baseline (Figure 6A), Crry was polarized to the basolateral aspect of tubules, and sporadic patches of $\mathrm{C} 3$ were seen along the tubular basement membrane. Little colocalization (yellow) was seen.

In order to quantitate colocalization of Crry and C3, we performed digital microscopy and measured the color intensity at each wavelength (Figure 6, D and E). Pixels were plotted according to their Crry and $\mathrm{C} 3$ intensity, and the number of pixels within the image that had a given staining intensity was indicated by color. A histogram of Crry and C3 intensity (Figure 6D) showed that there was virtually no colocalization of C3 and Crry. Pixels with a high intensity of both markers would map to the upper right quadrant. Very few pixels were present within this quadrant, indicating that those pixels with a high intensity for Crry staining did not have abundant C3. After 24 hours of reperfusion (Figure 6, C and E) there was much greater overall $\mathrm{C} 3$ deposition than at baseline, but there was still little, if any, colocalization of C3 and Crry. Renal I/R injury is patchy in nature, and some tubules retained their polarity (Figure $6 \mathrm{C}$, arrows) even in areas of extensive injury. Consistent with the histogram in Figure 6E, those tubules that retained the polarity of Crry showed little C3 deposition, whereas those with more severely altered Crry localization showed extensive C3 deposition.

Our results demonstrate that Crry localization was altered during renal ischemia prior to complement deposition and that tubules that retained Crry expression showed little C3 deposition. These data suggest that altered Crry localization is permissive of complement activation and that altered complement inhibition is a necessary event in the activation of complement after renal I/R. Although overall expression of Crry in the kidney did not significantly decrease (Figure 4), altered expression of Crry specifically on the surface of the tubules appeared to permit complement activation at that specific location. 

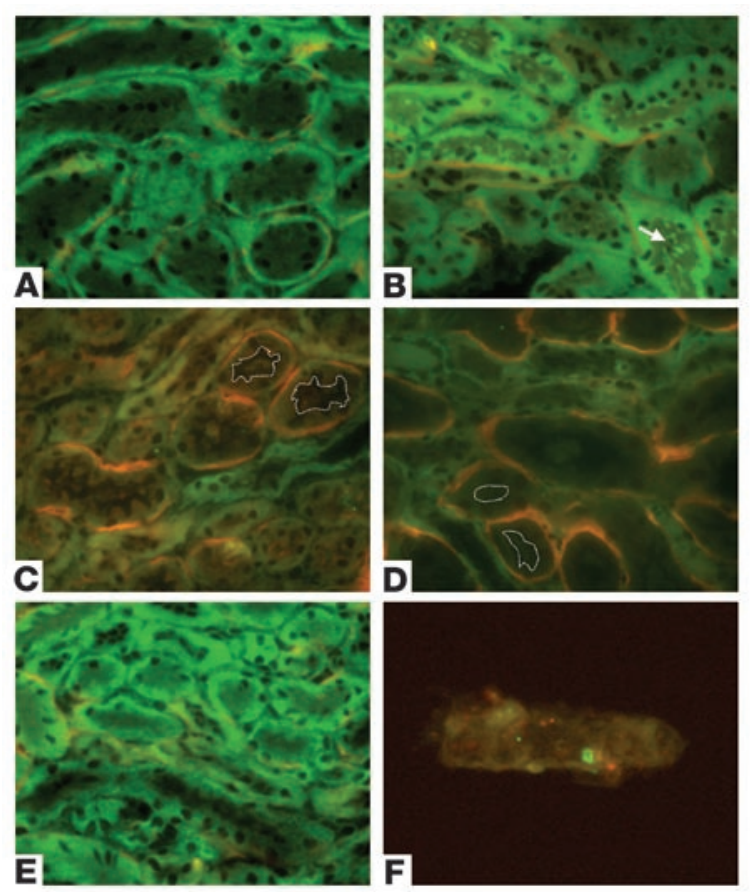

Altered Crry localization occurs primarily in the proximal tubule. Dual staining of kidney sections for Crry and a distal tubule marker revealed that altered Crry localization occurred primarily in proximal tubules (Figure 7A). It has long been known that $\mathrm{Na} / \mathrm{K}$-ATPase, another protein polarized to the basolateral aspect of PTECs, is associated with the actin cytoskeleton. Many studies have shown that I/R causes $\mathrm{Na} / \mathrm{K}$-ATPase to lose its polarity, and that after I/R the protein is seen diffusely throughout the cell cytoplasm (25, 26). Dual staining for Crry and $\mathrm{Na} / \mathrm{K}$-ATPase demonstrated that

\section{Figure 5}

The polarity of Crry is lost after I/R. Immunofluorescence microcopy was performed on tissue sections at baseline or after ischemia and variable times of reperfusion. (A-E) Dual staining for Crry (green) and C3 (red) was performed. (A) At baseline Crry was polarized to the basolateral aspect of the tubules in the kidney and scant C3 deposition was seen. (B) After 24 minutes of ischemia, the polarity of Crry expressed by proximal tubules was lost. Crry was seen more diffusely throughout the PTEC and within the tubule lumen (arrow). After 6 (C) and 24 hours (D) of reperfusion, an increasing percentage of tubules in the outer medulla ceased to express Crry. Those tubules that no longer expressed Crry showed extensive C3 deposition along their tubular basement membranes. Dashed lines indicate the tubule lumen, demonstrating that the tubule was still lined by epithelial cells although Crry was not detectable. (E) By 48 hours of reperfusion, Crry expression and the polarity of Crry was restored to baseline. (F) Staining of urinary sediment for F-actin (red) and Crry (green) revealed that after 2 hours of reperfusion, the sediment contained cellular blebs (as evidenced by the F-actin) and Crry. Original magnification, $\times 400$.

these proteins colocalized to the basolateral membrane of proximal tubules at baseline (Figure 7B). After I/R both shifted into the cytoplasmic domain. Colocalization of the proteins was weaker after I/R, however, suggesting distinct subcellular localizations.

Crry shifts from a triton-insoluble to a triton-soluble form after $I / R$. The polarity of many transmembrane proteins is maintained by attachment to the actin cytoskeleton (27), an association that can render a protein triton insoluble. A change in a renal tubular protein's solubility in triton after I/R may therefore reflect the disruption of this cytoskeletal attachment (25). To explore the possibility that tubular Crry is attached to the cytoskeleton, we examined the triton solubility of Crry before and after I/R. At baseline, approximately half of the $53-\mathrm{kDa}$ Crry isoform was present in the triton-extractable fraction of whole-kidney sections (Figure 7C). After I/R, however, nearly all of the protein was present in the triton-soluble fraction. The

\section{Figure 6}

Complement deposition after I/R does not colocalize with Crry. (A-C) Lower-powered views (original magnification, $\times 100)$ of the kidney demonstrate that the deposition of C3 during reperfusion only occurred along tubules that lost basolateral expression of Crry. Glomeruli (arrowheads) indicate cortical tissue, and the outer medulla is shown toward the bottom of each frame. (A) At baseline, Crry was heavily expressed in the glomerulus and along the basolateral aspect of the tubules. (B) After 2 hours of reperfusion, the polarity of Crry in many tubules was disrupted. (C) After 24 hours of reperfusion, most of the tubules had diminished levels of Crry. C3 was extensively deposited along the proximal tubules. Some tubules retained normal Crry expression and polarization (arrows) and showed little C3 deposition. In areas of heavy C3 deposition, little colocalization of C3 and Crry (yellow) is seen. (D and E) Histograms of Crry and C3 staining at baseline (D) and after 24 hours of reperfusion (E) further demonstrate that little colocalization occurred. Pixels are mapped according to Crry and C3 intensity. (D) The heat map shows the relative population densities depicted by color. Pixels with a high intensity of both $\mathrm{C} 3$ and Crry are plotted in the upper right quadrant. (E) Even after 24 hours of reperfusion, when the population of pixels with a high $\mathrm{C} 3$ intensity was much increased, virtually none of these high C3 pixels demonstrated concurrent high-intensity staining for Crry.
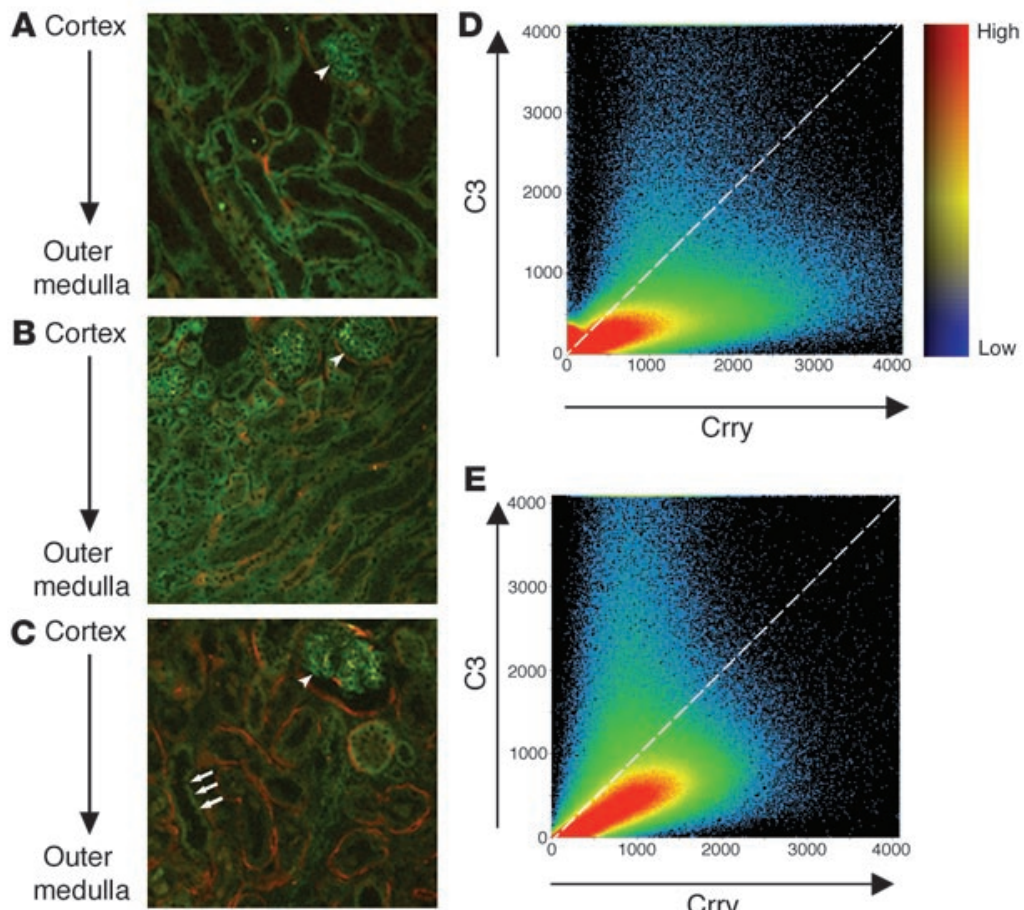

E

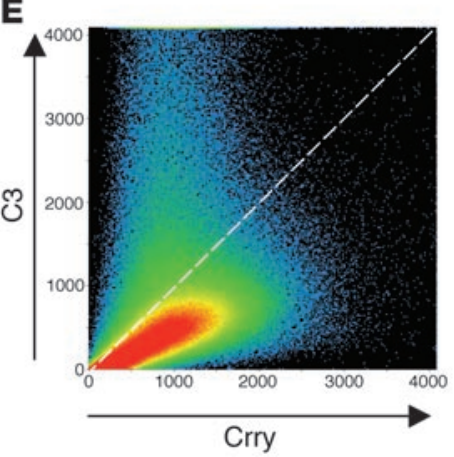


A
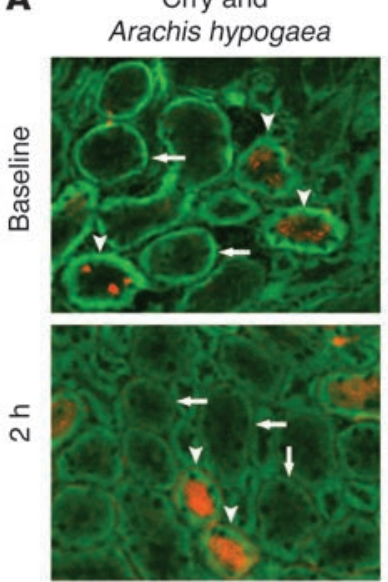

B
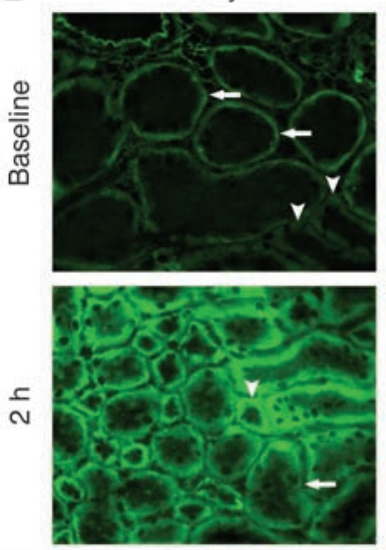

C

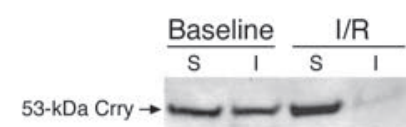

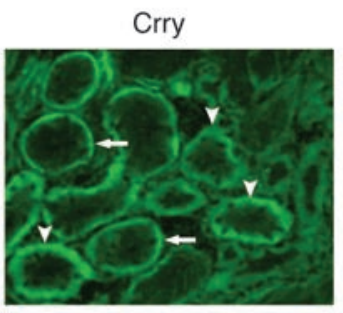
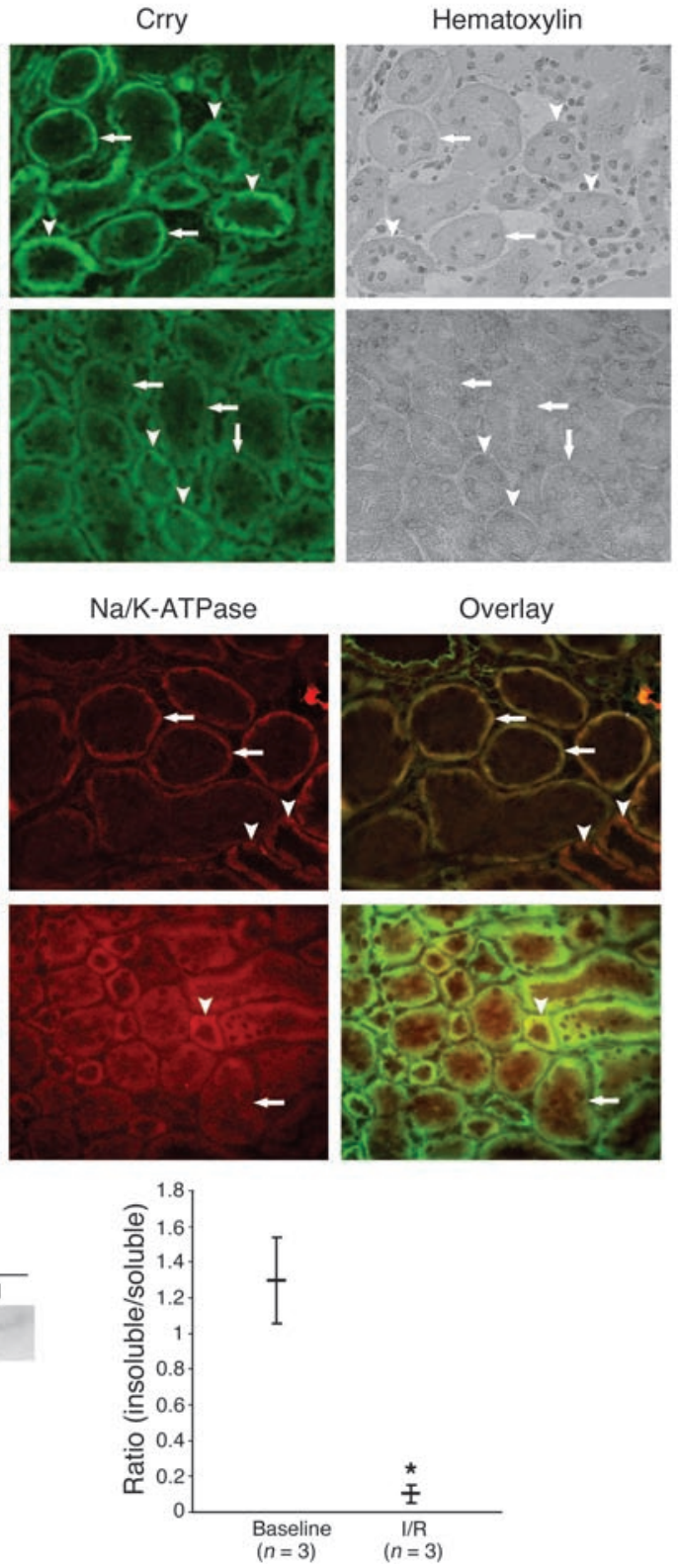

\section{Figure 7}

Loss of polarity in the proximal tubules after I/R. (A) Dual staining for Crry (green) and Arachis hypogaea (red, a marker of distal tubules) showed that Crry in proximal tubules had a sharp, basolateral localization (arrows), whereas expression in distal tubules (arrowheads) had a more diffuse appearance. After ischemia and 2 hours of reperfusion, the most prominent change was the loss of polarity in the proximal tubules. (B) Dual staining for Crry (green) and $\mathrm{Na} / \mathrm{K}$-ATPase (red) demonstrated that both were polarized proteins that colocalized at baseline to the basolateral aspect of proximal tubules (yellow). After ischemia and 2 hours of reperfusion the polarity of both proteins was lost, and both appeared dispersed throughout the cytoplasmic domain. (C) Protein was extracted from kidneys at baseline and after ischemia and 2 hours of reperfusion using a Triton $\mathrm{X}-100-$ containing lysis buffer. Fractions of triton-soluble (S) and -insoluble (I) protein were separated by SDS-PAGE and blotted for Crry. The 53-kDa form of Crry was detectable in the triton-insoluble fraction at baseline, and the ratio of triton-insoluble to -soluble Crry was greater than 1 . After ischemia and 2 hours of reperfusion, over $90 \%$ of the Crry had become triton soluble. ${ }^{*} P<0.01$ versus baseline. Original magnification, $\times 200$.

mice expressing less Crry at this location in the first place are more susceptible to complement activation. When subjected to 20 minutes of ischemia and 24 hours of reperfusion, $\mathrm{Crry}^{+/-}$mice had significantly greater SUNs $(111 \pm 18$ versus $52 \pm 8 \mathrm{mg} / \mathrm{dl} ; P<0.01)$ and significantly greater morphological injury (average ATN score, $3.9 \pm 0.3$ versus $2.56 \pm 0.6 ; P<0.05$ ) than did wild-type controls. Thus, mice that express decreased levels of Crry develop more severe injury in response to a mild ischemic insult.

Crry is critical for protecting PTECs in culture from alternative pathway-mediated injury. Complement activation may injure the tubular epithelium directly or through indirect effects such as alterations to the vasculature or chemotaxis of inflammatory cells. In order to confirm that PTECs themselves are the direct target of local alternative

other isoforms, in contrast, were primarily triton insoluble in both nonischemic and postischemic samples. This supports the hypothesis that the polarization of 53-kDa Crry in tubular epithelial cells is dependent upon attachment to the cytoskeleton, and the disruption of this attachment explains the loss of polarity seen after I/R.

Deficiency of Crry renders mice susceptible to renal I/R injury. To confirm that the loss of inhibition by Crry is an important event in the pathogenesis of ischemic ARF and complement activation, we subjected Crry-deficient and wild-type mice to a milder renal insult (Figure 8). $\mathrm{Crry}^{+/-}$mice do not directly address the issue of redistribution per se. They do, however, address the issue of the "concentration" of the complement inhibitor at the site of activation. We hypothesized that loss of functional Crry at the basolateral tubule would render the tubules more susceptible to injury, whether due to redistribution, targeted gene deletion, or the exogenous administration of an inhibitor of Crry (28). We therefore predicted that pathway-mediated injury when Crry function is impaired, we developed an in vitro model of PTEC injury. We incubated PTECs with fresh mouse serum in a buffer solution containing $10 \mathrm{mM}$ EGTA in order to inhibit classical pathway activation. Treatment of these cells with an inhibitory rat $\operatorname{IgG}_{1} \mathrm{mAb}$ to Crry in order to suppress the function of this protein caused injury to the cells as assessed by lactate dehydrogenase (LDH) release (Figure 9), whereas the addition of control rat $\operatorname{IgG}_{1}$ had no effect. Similar injury was also seen when Fab fragments of the inhibitory antibody were used, indicating that the injury is not a nonspecific effect of antibody binding or Fc-mediated complement activation. We then added the same inhibitor to a reaction mix in which the alternative complement pathway was selectively blocked with a mAb to factor B. Without an intact alternative pathway, blocking the function of Crry did not result in injury to the cells. Thus, functional Crry on the surface of PTECs is essential to the prevention of alternative pathway-mediat- 
A
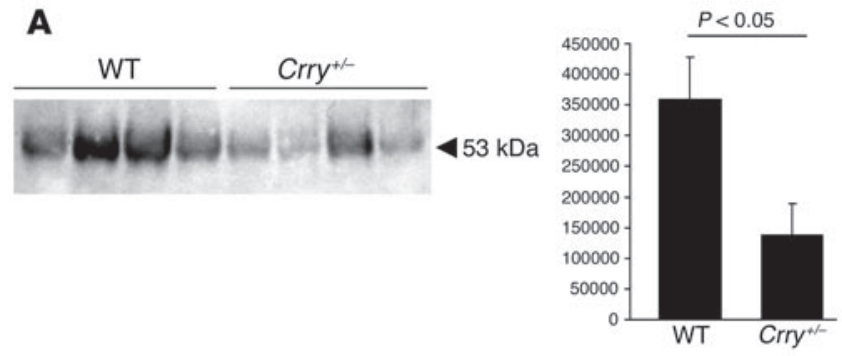

B
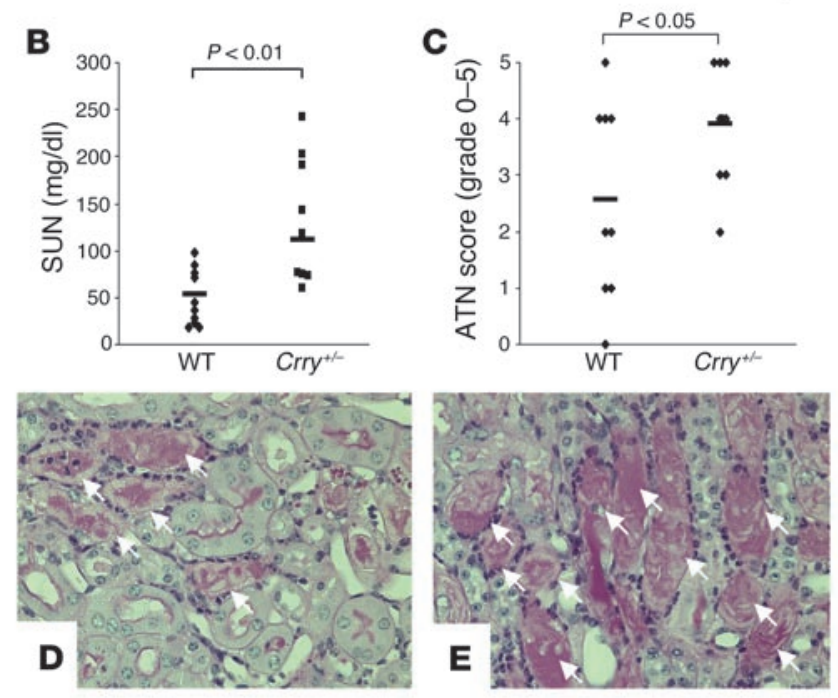

ed cellular injury. As described above, Crry was the only membranebound inhibitor of complement expressed by PTECs. The loss of complement inhibition by Crry on the surface of PTECs, whether by the application of an inhibitor of this protein in vitro or by the altered expression of Crry after ischemia, is therefore sufficient to permit alternative pathway-mediated injury of the cells.

Local synthesis of alternative pathway components after $I / R$. In other models, local synthesis of complement by the kidney has been found to be an important cause of complement activation and tissue injury (14). The kidney has been shown capable of synthesizing $\mathrm{C} 3$, factor $\mathrm{B}$, and factor $\mathrm{H}(29,30)$, and renal $\mathrm{I} / \mathrm{R}$ in rats was demonstrated to increase renal C3 expression (31). To determine whether the complement activation that occurs after I/R is enhanced by local synthesis of alternative pathway substrates,

\section{Figure 8}

Crry-deficient mice are more susceptible to I/R injury than wild-type controls. (A) Kidney lysates from Crry+/- mice and wild-type controls were blotted for Crry, demonstrating that the $\mathrm{Crry}^{+/-}$mice expressed approximately half the protein of wild-type controls. Crry+l- mice showed significantly greater increases in SUN (B) and morphological injury (C) compared with control mice after 20 minutes of ischemia and 24 hours of reperfusion. (D and E) Representative images from the outer stripe of the outer medulla in wild-type (D) and Crry ${ }^{+-}$(E) mice after I/R demonstrated that tubular injury (arrows) was more extensive in the $\mathrm{Crry}^{+/-}$mice. Original magnification, $\times 400$.

mice were subjected to sham surgery or to I/R. After variable times of reperfusion, the outer medulla (where $\mathrm{C} 3$ deposition is heaviest) was dissected out, and real-time PCR was performed to quantitate expression of C3, factor B, and factor H (Figure 10). C3 mRNA did not significantly increase at any of the times of reperfusion, although at 24 hours there was a trend toward increased C3 mRNA. Because of the tendency toward increased C3 synthesis after I/R, we more specifically evaluated the tubules of the outer medulla for changes in C3 synthesis using in situ hybridization (Figure 10). Local synthesis of C3 was evident in the outer medullas of sham-treated animals, and synthesis in this region increased after 24 hours of reperfusion. Factor H mRNA in the outer medulla was significantly decreased from baseline after 2 and 24 hours of reperfusion, as determined by real-time PCR. Thus increased local synthesis of C3 and decreased synthesis of factor H may further facilitate alternative pathway activation during reperfusion. Given the basal synthesis of $\mathrm{C} 3$ in this region of the kidney, it is also possible that a pool of locally produced $\mathrm{C} 3$ contributes to the activation that occurs after I/R. Furthermore, even small increases in C3 synthesis may serve to initiate a much larger degree of complement activation, subsequently utilizing circulating components of the alternative pathway.

\section{Discussion}

Complement activation by the alternative complement pathway is an important pathogenic event after I/R $(1,2)$. C3 deposition primarily occurs along the tubular basement membrane of proximal tubules in the outer stripe of the outer medulla, and Crry is the only membrane-bound inhibitor of complement expressed by the tubular epithelial cells in this segment of the nephron. At baseline, Crry is polarized to the basolateral aspect of the tubules, adjacent to where complement deposition occurs after I/R. Mice that are

\section{Figure 9}

Intact Crry function is necessary to prevent injury to PTECs by alternative pathway components present in mouse serum. PTECs were incubated with fresh mouse serum in the presence or absence of 5D5, an inhibitory mAb to Crry. When incubated with 5D5, the addition of serum to the PTECs resulted in $\mathrm{LDH}$ release by the cells. The addition of control IgG, in contrast, did not injure the cells. The addition of the $m A b 1379$, a specific inhibitor for factor B of the alternative pathway, prevented the release of LDH by cells incubated with 5D5 and serum. The reactions were conducted with $10 \mathrm{mM}$ EGTA to prevent activation of the classical pathway by the antibody. The experiment was repeated using IgG and Fab fragments of the 5D5 antibody, and LDH release was similar in the 2 groups.
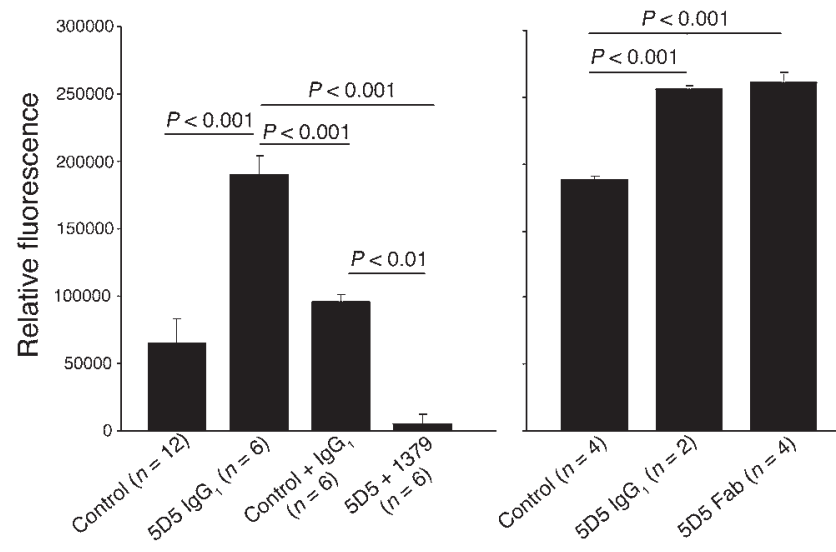


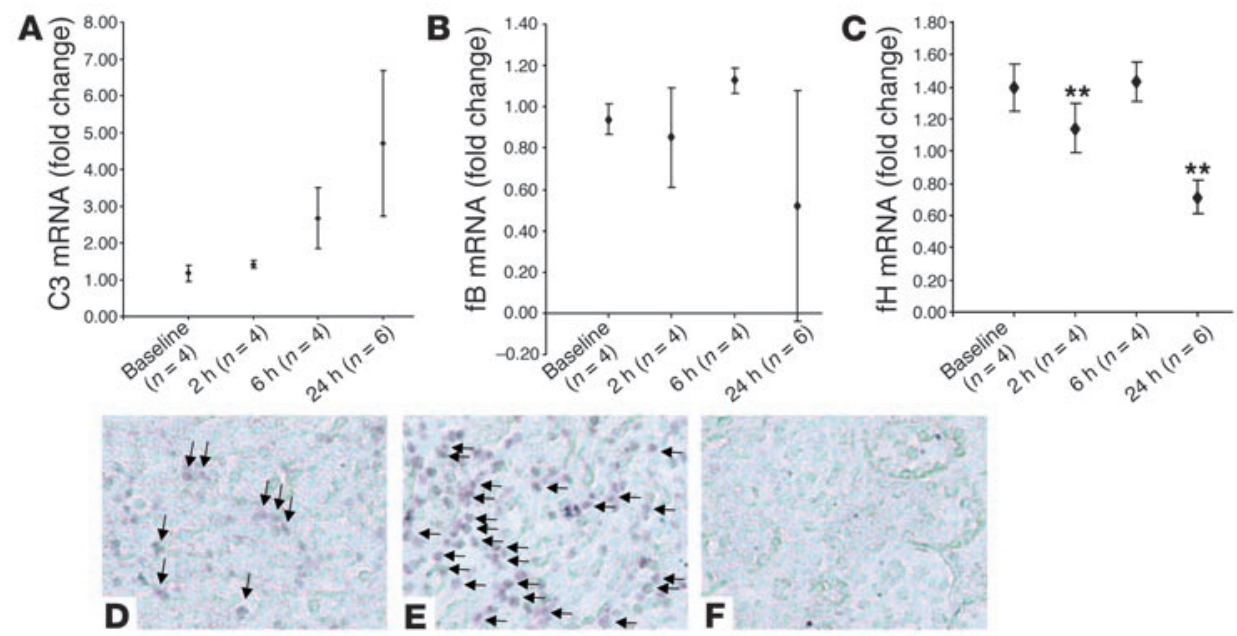

Figure 10

Local synthesis of complement components within the kidney after I/R. The outer medulla was dissected out from unmanipulated kidneys and kidneys subjected to ischemia and variable times of reperfusion. mRNA was isolated from these samples, and quantitative real-time PCR was performed to assess changes in the local synthesis of the complement components $\mathrm{C} 3(\mathbf{A})$, factor $\mathrm{B}(\mathrm{fB}, \mathrm{B})$, or factor $\mathrm{H}(\mathrm{fH}, \mathrm{C})$ after I/R. These components were expressed at low levels within unmanipulated kidneys. A trend toward increased C3 was seen after 24 hours but did not reach statistical significance. Significant decreases in factor $\mathrm{H}$ synthesis were observed after 2 and 24 hours of reperfusion $\left({ }^{* \star} P<0.01\right.$ versus sham treatment). ( $\mathbf{D}$ and $\left.\mathbf{E}\right)$ In situ hybridization for $\mathrm{C} 3$ demonstrated that $\mathrm{C} 3$ mRNA (arrows) was detectable in the outer medulla of sham-treated animals (D), but levels were much higher after ischemia and 24 hours of reperfusion (E). Hybridization of sham (not shown) and postischemic (F) kidneys with a sense probe for C3 were performed as negative controls. In situ hybridization was performed on 4 animals per group, and representative images are shown.

deficient in DAF and CD59 are susceptible to complement-mediated injury after renal I/R $(19,20)$, likely due to complement activation along the vascular endothelium (19). However, extensive complement activation after $\mathrm{I} / \mathrm{R}$ in wild-type mice occurs along the tubular epithelium. Thus, I/R must cause the Crry expressed by these cells to become overwhelmed.

We have demonstrated herein that ischemia quickly led to altered localization of Crry. Even before reperfusion of the kidney began, Crry shifted away from the basolateral membrane and into the cytoplasm. Although levels of Crry in the whole kidney remained close to baseline after I/R, immunofluorescence demonstrated that levels of the protein in proximal tubules in the outer stripe of the outer medulla were much lower within 6 hours of reperfusion, and Crrycontaining blebs appeared in the urine after I/R. A trend toward increased Crry mRNA in the outer medulla suggests that replacement of the lost Crry by the repaired kidney is accomplished by synthesis of new protein. Complement deposition along the tubules occurred only after the loss of Crry at this location. Despite abundant C3 deposition after I/R there was little colocalization of C3 and Crry, suggesting that the loss of Crry at the basolateral surface is necessary for activation of the alternative pathway. Conversely, those tubules that retained polarized expression of Crry along their surface demonstrated little C3 deposition.

Crry is analogous to other polarized membrane-associated proteins such as $\mathrm{Na} / \mathrm{K}$-ATPase, which has long been known to lose its polarity during ischemia (25). Like $\mathrm{Na} / \mathrm{K}$-ATPase, Crry within the kidney also shifted from a triton-insoluble fraction to an almost entirely triton-soluble state during ischemia. As is the case with $\mathrm{Na} / \mathrm{K}$-ATPase, this biochemical shift of Crry probably reflects the loss of a stabilizing association, such as with the actin cytoskeleton. The rapid change in the triton solubility of Crry and movement of it away from the basolateral membrane preceded detectable activation of complement on the tubular cells.

Treatment with an inhibitor of Crry leads to increased complement activation and spontaneous tubular injury in rats (28). Furthermore, we have found that mice expressing lower levels of Crry (Crry ${ }^{+/}$mice) were more susceptible to renal I/R. This supports the hypothesis that a decreased concentration of Crry at the basolateral aspect of the PTEC (whether due to a targeted gene mutation, the administration of an exogenous inhibitor, or altered expression in response to $\mathrm{I} / \mathrm{R}$ ) leaves the tubules more susceptible to complement-mediated injury. When the function of Crry expressed by PTECs in culture was blocked, the cells were injured in the presence of alternative pathway-sufficient serum. Blocking the function of Crry did not injure PTECs when the alternative complement pathway in the serum to which they were exposed was also inhibited. This confirms the importance of Crry for the prevention of autologous complement-mediated tubular injury. Loss of this protection, due to either congenital deficiency, exogenous inhibition, or loss of polarity after I/R, permits uncontrolled activation of the alternative pathway on the surface of tubular epithelial cells. A study in which exogenous Crry-Ig was administered to mice before they underwent $\mathrm{I} / \mathrm{R}$ did not find that this agent significantly protected the mice from ARF (32). All of the mice in this study were also treated with heparin, however, and heparin is a very effective complement inhibitor (33). It is therefore likely that heparin inhibited complement activation in both experimental groups, obscuring any potential benefit derived from Crry-Ig.

Previous studies have shown that PTECs in culture activate the alternative pathway when they are heat killed (34), and that such activation results in an inflammatory response by the cells (35). It is possible that altered expression of Crry is a general response to injury of these cells, and that complement activation by tubular epithelial cells also occurs in other forms of tubular injury. In support of this, we have observed that glycerol-induced rhabdomyolysis (a model of toxin-induced ATN) is also characterized by decreased tubular Crry and peritubular C3 deposition (our unpublished observations).

We have examined the effects of $\mathrm{I} / \mathrm{R}$ on the intrinsic inhibitors of complement activity and on local synthesis of the complement components. Several previous studies have highlighted the contribution of locally synthesized C3 to the development of renal injury $(14,31)$. Real-time PCR of samples made from the outer medulla demonstrated that there was basal synthesis of $\mathrm{C} 3$ in this region, and synthesis of C3 was increased after $\mathrm{I} / \mathrm{R}$ when evaluated 
Table 1

Gene-specific primers and probes

$\begin{array}{ll}\text { Primer } & \text { Sequence } \\ \text { C3 for } & \text { 5'-GGAAACGGTGGTGGAGAAAGC-3' } \\ \text { C3 rev } & \text { 5'-CTCTTGACAGGAATGCCATCGG-3' } \\ \text { Factor B for } & \text { 5'-CTCGGGCTCCATGAATATCTACC-3' } \\ \text { Factor B rev } & \text { 5'-CCGTAACTCGCCACCTTCTC-3' } \\ \text { Factor H for } & \text { 5'-GAGCCTGAGACCCAACTTCC-3' }^{\prime} \\ \text { Factor H rev } & \text { 5'-CTGTGCAACGAAGGACTCC-3' } \\ \text { Cyclophilin for } & \text { 5'-TGGAGAGCACCAAGACAGACA-3' } \\ \text { Cyclophilin rev } & \text { 5'-TGCCGGAGTCGACAATGAT-3' } \\ \text { Crry for } & \text { 5'-AGTGGATTCCTTGTGAGATACCC-3' } \\ \text { Crry rev } & \text { 5'-TTGAGTTCAATGCACTGAGGAGG-3' } \\ \text { GAPDH for } & \text { 5'-CCATGGAGAAGGCCGGG-3' } \\ \text { GAPDH rev } & \text { 5'-CAAAGTTGTCATGGATGACC-3' } \\ \text { C3 antisense probe } & \text { 5'-GTCGATGGCGATGAGGTTGGCAGCTAGAGAGAAGACCTTGACCACGTA-3' }\end{array}$

for, forward; rev, reverse.

Membrane cofactor protein (MCP, also known as CD46), a human functional analog of Crry, is expressed by human PTECs in a polarized fashion (38). Studies have demonstrated that the polarity of PTECs is disrupted in human ATN (39). Loss of polarity of MCP after I/R may also therefore occur, permitting activation of the alternative pathway.

Our results may also have broader implications regarding the regulation of complement activation. The importance of the complement system in the pathogenesis of immune complex diseases as well as the mechanisms of complement activation in these diseases are well established. The complement system has recently been implicated in a large number of Ig-independent diseases (40-42), however, and the cause of activation in these diseases has yet to be fully

by in situ hybridization. In contrast, local synthesis of factor $\mathrm{H}$ was decreased after I/R. Thus, I/R causes a shift toward greater C3 production and decreased factor $\mathrm{H}$ synthesis in the outer medulla during the period when Crry expression is most disrupted.

It is possible that $\mathrm{I} / \mathrm{R}$ of the kidney results in the generation of specific alternative pathway activators. Moieties such as endotoxin, IgA, ammonia, and nephritic factor can cause activation of the alternative pathway. We have conducted experiments in which mice were fed water containing potassium bicarbonate or ammonium chloride in an effort to alter the renal ammonia content and therefore alter the magnitude of complement activation after $\mathrm{I} / \mathrm{R}$. These manipulations did not detectably affect the degree of complement activation in the kidney either before or after I/R (our unpublished observations). Although the presence of ammonia may be an important component of peritubular alternative pathway activation, changes in local ammonia content do not explain the increase in complement activation seen after I/R. There are no obvious mechanisms for accumulation of the other known alternative pathway activators in this model, but we have not excluded the possibility that ischemic tubular epithelial cells generate and release proteases that could contribute to this process.

The polarization of proteins within renal epithelia is essential to the proper function of the kidney. Molitoris et al. (36) demonstrated that the basolateral polarization of $\mathrm{Na} / \mathrm{K}$-ATPase is lost after I/R. Disruption of the polarized organization of proteins within the plasma cell membrane results in the loss of vectorial transport across the epithelium, loss of tight junctions between epithelial cells, and loss of integrin-mediated adhesion of epithelial cells to the extracellular matrix (37). Our results demonstrate that the loss of polarized expression of Crry renders the epithelial cells prone to complement activation and further injury. Furthermore, the generation of proinflammatory complement activation products such as the anaphylatoxin C5a or the generation of other proinflammatory cytokines caused by uncontrolled complement activation along the tubular epithelium (35) may have widespread and deleterious consequences for the host. Insults to the precise organization of the tubular epithelium therefore impair renal function as well as contribute to the local, and possibly systemic, inflammatory response.

We previously found that human ATN is also characterized by alternative pathway activation along the proximal tubule (15). elucidated. Although genetic complement deficiencies are known to cause autoimmune disease $(10,13)$, nongenetically determined loss of complement inhibition of a specific organ or cell type may significantly contribute to inflammation and organ injury. Studies in rats have shown that CD59 expression in the myocardium decreases after ischemia, perhaps allowing greater assembly of the membrane attack complex (43), although in that setting complement activation precedes loss of inhibition. Anoxic injury of human endothelial cells in culture also results in diminished expression of cell surface complement inhibitors and activation of the complement system on the cell surface (44). Given the need for constant inhibition of the alternative pathway to prevent spontaneous injury, it is possible that other injuries in various tissues may impede this tonic inhibition and result in alternative pathway activation. Locations such as the renal tubules that rely on only 1 complement inhibitor may be particularly vulnerable to such injuries.

We have demonstrated that altered localization and expression of renal tubular epithelial Crry is an early consequence of renal ischemia. The loss of inhibition by Crry is permissive of complement activation on the surface of epithelial cells and of the resultant complement-mediated injury. These results explain the unique nature of complement activation in the kidney after I/R. Furthermore, these findings demonstrate that complement activation may be a response to any injury that alters host expression of complement inhibitors. Inhibition of the alternative pathway may be an effective means of ameliorating ischemic ARF. The loss of complement inhibition and the activation of the alternative pathway are early events after I/R, however, and exogenous inhibition may need to be performed soon after the ischemic event. The exact localization of cell surface complement inhibitors is clearly very important, and exogenous inhibitors of complement may not effectively prevent activation of complement on the epithelial cell surface if they do not achieve adequate levels specifically at this site.

\section{Methods}

Experimental animals. C57BL/6J mice (The Jackson Laboratory) were used as wild-type mice for experiments examining the relationship of Crry expression and complement activation. Crry-deficient mice were generated as previously described (45). Mice that were heterozygous for Crry $\left(\mathrm{Crry}^{+/-}\right)$, littermate controls, and $\mathrm{C} 57 \mathrm{BL} / 6 \mathrm{~J}$ mice were used to test the 
effects of Crry deficiency since homozygous deficiency is lethal in utero (45). Only male mice were used in this study because they demonstrate a higher level of complement activity and are more vulnerable to complement-mediated renal injury (32). Sprague-Dawley rats were used for isolation of renal basolateral and brush border membranes and for immunogold labeling. The animals were housed and maintained in the University of Colorado Center for Laboratory Animal Care in accordance with the NIH Guidelines for the Care and Use of Laboratory Animals. The study protocol was approved by the University of Colorado Health Sciences Center Animal Care and Use Committee.

Antibodies and primers. The following antibodies were used for immunofluorescence: FITC-conjugated goat anti-mouse C3 (Cappel; MP Biomedicals) diluted 1:50 with PBS, rat anti-mouse Crry (BD Biosciences Pharmingen) diluted 1:150, rabbit anti-human C3d (Dako) diluted 1:150, mouse anti- $\mathrm{Na} / \mathrm{K}$-ATPase $\alpha-1$ (Upstate USA Inc.) at a concentration of $0.5 \mu \mathrm{g} / \mathrm{ml}$, rabbit anti-mouse collagen type IV (Chemicon International) diluted 1:400. FITC-conjugated Arachis bypogaea (Sigma-Aldrich) was used as a distal tubule marker (46). A polyclonal rabbit anti-DAF antibody and a mouse anti-CD59 mAb (a generous gift of S. Tomlinson, Medical University of South Carolina, Charleston, South Carolina, USA) were diluted 1:300. To stain F-actin, Alexa Fluor 350 phalloidin (Invitrogen Corp.) was diluted 1:40. For secondary antibodies, FITC-conjugated anti-rat IgG, rhodamine-conjugated anti-rabbit IgG, and FITC-conjugated anti-mouse IgG (Cappel; MP Biomedicals) were used at dilutions of 1:100, and Alexa Fluor 488-conjugated goat anti-rabbit IgG was used at a dilution of 1:400 (Invitrogen Corp.). Two mAbs were used as complement protein inhibitors. The mAb 5D5 (47) was used to inhibit the function of cell-surface Crry, and $\mathrm{mAb} 1379$ (48) was used to block mouse alternative pathway activity. Fab fragments of 5D5 were also made using papain-agarose (MP Biomedicals) according to the manufacturer's instructions.

For Western blot analysis, a peroxidase-conjugated goat anti-mouse C3 (Cappel; MP Biomedicals) was used at a dilution of $0.3 \mu \mathrm{g} / \mathrm{ml}$. A polyclonal antibody to Crry (23) and a polyclonal anti-type IIa $\mathrm{Na} / \mathrm{Pi}$ cotransporter antibody (49) were used at dilutions of $1 \mu \mathrm{g} / \mathrm{ml}$, followed by incubation with peroxidase-conjugated goat anti-rabbit IgG (Sigma-Aldrich).

Induction of ischemic ARF. Mice weighing 20-25 g were anesthetized with $300 \mu \mathrm{l}$ 2,2,2-Tribromoethanol (Sigma-Aldrich) injected intraperitoneally. After the mice were anesthetized they were placed on a heating pad to maintain their body temperature during surgery. Laparotomies were then performed, and the renal pedicles were located and isolated by blunt dissection. The pedicles were clamped with surgical clips (Miltex Inc.), and occlusion of blood flow was confirmed by visual inspection of the kidneys. The clamps were left in place for 24 minutes and then released. This time of ischemia was chosen to obtain a reversible model of ischemic ARF with a minimum of vascular thrombosis and to avoid animal mortality. For experiments comparing $\mathrm{Crry}^{+/-}$mice with $\mathrm{Crry}^{+/+}$controls, an ischemic time of 20 minutes was used. The shorter ischemic time was chosen because it causes milder injury, allowing for detection of mouse strains with greater susceptibility to I/R injury. The kidneys were observed for approximately 1 minute to ensure blood reflow, then fascia and skin were sutured with 4-0 silk (U.S. Surgical). Sham surgery was performed in an identical fashion, except that the renal pedicles were not clamped. The mice were volume resuscitated with $0.5 \mathrm{ml}$ of normal saline and kept in an incubator at $29^{\circ} \mathrm{C}$ to maintain body temperature. After $0,2,6,24$, or 48 hours of reperfusion the mice were anesthetized, and blood was obtained by cardiac puncture. Laparotomy was performed and the kidneys were harvested.

SUN measurements. SUN was determined for each mouse using a Beckman Autoanalyzer (Beckman Coulter).

Renal morphology. After the kidneys were removed from the mice, sagittal sections were fixed in $4 \%$ paraformaldehyde. After being embedded in paraffin, $4-\mu \mathrm{m}$ sections were cut and stained with periodic acid-Schiff. The sections were evaluated by a renal pathologist (D. Ljubanović) in a blinded fashion. The cortex and outer stripe of the outer medulla were assessed for epithelial necrosis, loss of brush border, tubular dilatation, and cast formation. At least 10 fields (magnification, $\times 400$ ) were reviewed for each slide, and the percentage of tubules displaying these findings was determined. Kidney sections were scored as follows based on the percentage of affected tubules: 0 , none; 1 , less than $10 \%, 2,10-25 \%, 3,26-45 \%, 4,46-75 \%$, 5 , greater than $75 \%$.

Immunofluorescence and in situ bybridization. For immunofluorescence, sagittal sections of the kidneys were snap frozen in OCT compound (Sakura Finetek). Sections $(4 \mu \mathrm{m})$ were cut with a cryostat and stored at $-70^{\circ} \mathrm{C}$. The slides were later fixed with acetone and incubated with the primary antibodies. After hybridization with antibodies was complete the slides were counterstained with hematoxylin (Vector Laboratories). Tissue sections were incubated with secondary antibodies alone to ensure the specificity of antibody binding.

To detect the presence of Crry in the urine, immunofluorescence was performed on urine sediments as previously described (24). Upon sacrifice after 6 hours of renal reperfusion, urine was expressed from the bladder and collected at the tip of the urethra. Urine was centrifuged at $4{ }^{\circ} \mathrm{C}$ for 10 minutes at 15,996 g. The pellets were then fixed for 1 hour at room temperature in $3 \%$ paraformaldehyde in PBS ( $\mathrm{pH} 7.4$ ). The sediment was then washed 3 times in PBS for 5 minutes and recentrifuged for 5 minutes at $15,996 \mathrm{~g}$. The pellets were incubated for 10 minutes in $0.1 \%$ Triton X-100 (Sigma-Aldrich) in PBS. Nonspecific binding was blocked with 2\% BSA in PBS for 1 hour. The pellets were then incubated with rat anti-mouse Crry and Alexa Fluor 350 phalloidin. To ensure that antibody binding was specific, anti-secondary antibody alone was used as a control.

Imaging was performed on a Nikon T2000 inverted fluorescent microscope and slidebook software (version 3.0; Intelligent Imaging Innovations Inc.). Intensity histograms were obtained using a Zeiss LSM 510 Meta confocal microscope running Zeiss software version 3.2 Service Pack 2.

To examine kidneys for the local production of C3, 16- $\mu \mathrm{m}$ kidney sections were fixed for 15 minutes in cold $4 \%$ paraformaldehyde. The sections were then hybridized with a digoxin-labeled antisense probe to mouse $\mathrm{C} 3$ (Table 1) according to the manufacturer's instructions (GeneDetect.com). The bound probe was detected using alkaline phosphatase-labeled antidigoxigenin Fab fragments and NBT/BCIP tablets (Roche Diagnostics Corp.). Digoxin-labeled sense probe to mouse $\mathrm{C} 3$ was used to confirm the specificity of the binding.

Immunogold labeling of Crry. Rat kidneys were fixed by perfusion with $2 \%$ formaldehyde and $0.1 \%$ glutaraldehyde in $0.1 \mathrm{M}$ sodium cacodylate, $\mathrm{pH}$ 7.35 , as previously described (50). The samples were dehydrated and embedded in LR Gold resin (Polysciences Inc.). Ultrathin sections were then prepared using nickel grids and stained for Crry. The grids were floated on droplets of PBS (10 minutes), 10\% whole goat serum (10 minutes), and either droplets of rabbit anti-Crry at a dilution of $20 \mu \mathrm{g} / \mathrm{ml}$ or nonimmune rabbit serum diluted 1:150 (45 minutes). The grids were washed using droplets of PBS for a total of 20 minutes, after which each was floated on $10 \mu \mathrm{l}$ of goat anti-rabbit IgG Fab2 conjugated to 10 -nm gold particles (Ted Pella Inc.). After washing the grids with PBS, they were stained for 6 minutes with $2 \%$ uranyl acetate, washed with water, and air dried. The sections were then viewed using a Tecnai $\mathrm{G}^{2}$ microscope (FEI Co.). After identifying PTECs, images were taken at a magnification of $\times 18,500$ in the apical and basal regions of the cells, and the number of gold particles seen in each view were counted.

Western blot analysis. Renal tissue was homogenized in RIPA lysis buffer containing 1\% Triton X-100, 0.5\% deoxycholic acid, $150 \mathrm{mM} \mathrm{NaCl}, 20 \mathrm{mM}$ $\beta$-glycerophosphate, $20 \mathrm{mM}$ Tris- $\mathrm{HCl}$ ( $\mathrm{pH} 8.0$ ), 5 mM EGTA, $3 \mathrm{mM} \mathrm{MgCl}_{2}$, 
$0.1 \% \mathrm{SDS}, 1 \mathrm{mM} \mathrm{DTT}, 50 \mu \mathrm{M} \mathrm{Na}_{3} \mathrm{VO}_{4}$, and 1 tablet of complete, EDTA-free protease inhibitor cocktail (Roche Diagnostics Corp.). Homogenates were centrifuged at $15,996 \mathrm{~g}$ for 15 minutes at $4^{\circ} \mathrm{C}$, and the supernatant was collected. Brush border and basolateral membranes from rat renal cortical tissue were isolated as previously described (51). Briefly, kidneys were placed in cold isolation buffer containing $300 \mathrm{mM}$ mannitol, $5 \mathrm{mM}$ EGTA, 0.1 $\mathrm{mM}$ PMSF, and $18 \mathrm{mM}$ Tris ( $\mathrm{pH}$ 7.4). Thin cortical sections were obtained and homogenized. Brush border and basolateral membranes were then isolated by differential centrifugation, magnesium precipitation, and use of a discontinuous sucrose gradient (52). To isolate triton-soluble and -insoluble protein fractions, kidneys were homogenized in a solution containing $0.1 \%$ Triton X-100, $20 \mathrm{mM}$ HEPES, $120 \mathrm{mM} \mathrm{KCl}, 12 \mathrm{mM} \mathrm{NaCl}, 1.62 \mathrm{mM}$ $\mathrm{MgSO}_{4}, 1 \mathrm{mM}$ EDTA, $0.35 \mathrm{mM} \mathrm{CaCl}$, and 1 tablet of complete, EDTAfree protease inhibitor cocktail (Roche Diagnostics Corp.). Samples were centrifuged at $35,000 \mathrm{~g}$ for 14 minutes at $4^{\circ} \mathrm{C}$ to separate the soluble and insoluble fractions. Because approximately two-thirds of cellular proteins are triton soluble (53), the insoluble pellet was resuspended at half the volume of buffer used for the initial homogenization. Protein concentrations for all of the samples were determined using the Bio-Rad protein assay (Bio-Rad Laboratories). Protein $(50 \mu \mathrm{g})$ for each sample was resolved by electrophoresis with a $10 \%$ Bis-Tris polyacrylamide gel (Invitrogen Corp.) and transferred to a nitrocellulose membrane. The membrane was probed with antibody to Crry and visualized using a chemiluminescence detection kit (Amersham Biosciences).

Reverse transcription polymerase chain reaction. Kidneys were obtained from unmanipulated mice and mice subjected to ischemia and variable times of reperfusion. The outer medullary region of the kidney was manually dissected away from the inner medulla and the outer cortex, and RNA was isolated using TRIzol reagent (Gibco; Invitrogen Corp.). cDNA was generated from $3 \mu \mathrm{g}$ RNA using MuLV Reverse Transcriptase (Applied Biosystems). Quantitative real-time PCR for C3, factor B, and factor $\mathrm{H}$ was then performed using the designated primers (Table 1). Appropriate primers were designed using Beacon Designer software (version 4.0; PREMIER Biosoft International). Samples were prepared using iQ Sybergreen Supermix (Bio-Rad Laboratories) and the iCycler iQ detection system (Bio-Rad Laboratories). Triplicate reactions for each sample were performed using the target primers and using cyclophilin primers to provide an internal control. Conventional PCR for Crry was performed in a GeneAmp 9700 PCR system (Applied Biosystems) after using cDNA from an unmanipulated kidney to determine the linear range of amplification. Control PCR was performed using primers for glyceraldehyde3 -phosphate dehydrogenase. PCR products were run on $2 \%$ agarose gels and visualized with ethidium bromide.

In vitro assay of complement inhibition by Crry on PTECs. Boston University mouse proximal tubular cells (BUMPT cells) were generously provided to us by J. Schwartz (Boston University Medical Center, Boston, Massachusetts, USA). This is a mouse PTEC line that was established from the immortoMouse (54). Cells were grown to confluence in DMEM supplemented with $10 \%$ fetal bovine serum, penicillin/streptomycin, and 0.2 $\mathrm{U} / \mathrm{ml}$ IFN- $\gamma$ (PeproTech Inc.). After the cells reached confluence they were changed to 1:1 DMEM/Hams F12 supplemented with $5 \mathrm{mg} / \mathrm{l}$ transferrin (Invitrogen Corp.), $50 \mathrm{nM}$ hydrocortisone (Sigma-Aldrich), and $5 \mathrm{mg} / 1$ insulin for 2 days. As a source of complement components, $10 \%$ serum from C57BL/6J mice was added to a reaction mix containing $5 \mathrm{mM} \mathrm{MgCl} 2$ and $10 \mathrm{mM}$ EGTA in PBS. Some of the cells were also treated with 100 $\mu \mathrm{g} / \mathrm{ml} 5 \mathrm{D} 5$, an inhibitory mAb to Crry (47), or the same concentration of control rat $\mathrm{IgG}_{1}$ (Sigma-Aldrich). To confirm that the effects of 5D5 were due to its inhibitory activity and a nonspecific effect of antibody binding, $70 \mu \mathrm{g} 5 \mathrm{D} 5 \mathrm{Fab}$ were added to cells with serum and compared with cells treated with 5D5 IgG. Another group of cells was treated with 5D5 after preincubating the mouse serum with $40 \mu \mathrm{g} 1379$, an inhibitory mAb to mouse factor B (an essential component of the alternative pathway). Cells were incubated for 10 minutes, and supernatant was then tested for LDH content. LDH was measured by the Cytotox-ONE assay (Promega) according to the manufacturer's instructions, and the results were read on a Wallac 1420 fluorescence plate reader (PerkinElmer and Analytical Sciences Inc.) with an excitation wavelength of $530 \mathrm{~nm}$ and an emission wavelength of $590 \mathrm{~nm}$.

Statistics. Multiple-group comparisons were performed using ANOVA with post-test according to Newman-Keuls. Comparison between the control and $\mathrm{Crry}^{+/-}$groups was performed with the Mann-Whitney test. A $P$ value of less than 0.05 was considered statistically significant. Results are reported as mean \pm SEM.

\section{Acknowledgments}

These studies were supported by NIH grants R0-1 AI31105 (to V.M. Holers) and K08 DK064790-01 (to J.M. Thurman), a postdoctoral fellowship from the National Kidney Foundation (to J.M. Thurman), and a postdoctoral fellowship from the International Society of Nephrology (to D. Ljubanović). The authors would also like to thank Rolf Dahl for his assistance with the immunogold labeling.

Received for publication January 19, 2005, and accepted in revised form November 29, 2005.

Address correspondence to: Joshua M. Thurman, Division of Nephrology and Hypertension, B-115, University of Colorado Health Sciences Center, 4200 East 9th Avenue, Denver, Colorado 80262, USA. Phone: (303) 315-0171; Fax: (303) 315-5540; E-mail: Joshua.Thurman@UCHSC.edu.
1. Thurman, J.M., Ljubanovic, D., Edelstein, C.L., Gilkeson, G.S., and Holers, V.M. 2003. Lack of a functional alternative complement pathway ameliorates ischemic acute renal failure in mice. J. Immunol. 170:1517-1523.

2. Zhou, W., et al. 2000. Predominant role for C5b-9 in renal ischemia/reperfusion injury. J. Clin. Invest. 105:1363-1371.

3. De Vries, B., et al. 2003. Complement factor C5a mediates renal ischemia-reperfusion injury independent from neutrophils. J. Immunol. 170:3883-3889.

4. Pratt, J.R., et al. 2003. Nontransgenic hyperexpression of a complement regulator in donor kidney modulates transplant ischemia/reperfusion damage, acute rejection, and chronic nephropathy. Am. J. Pathol. 163:1457-1465.

5. Park, P., et al. 2002. Injury in renal ischemia-reperfusion is independent from immunoglobulins and T lymphocytes. Am. J. Physiol. Renal Physiol. 282:F352-F357.

6. Williams, J.P., et al. 1999. Intestinal reperfusion injury is mediated by IgM and complement. J. Appl. Physiol. 86:938-942.

7. Fleming, S.D., et al. 2002. Mice deficient in complement receptors 1 and 2 lack a tissue injury-inducing subset of the natural antibody repertoire. J. Immunol. 169:2126-2133.

8. Kagiyama, A., et al. 1989. Molecular basis of complement activation in ischemic myocardium: identification of specific molecules of mitochondrial origin that bind human $\mathrm{C} 1 \mathrm{q}$ and fix complement. Circ. Res. 64:607-615.

9. Liszewski, M.K., Farries, T.C., Lublin, D.M., Rooney, I.A., and Atkinson, J.P. 1996. Control of the complement system. Adv. Immunol. 61:201-283.

10. Pickering, M.C., et al. 2002. Uncontrolled C3 acti- vation causes membranoproliferative glomerulonephritis in mice deficient in complement factor H. Nat. Genet. 31:424-428.

11. Goodship, T.H., Liszewski, M.K., Kemp, E.J., Richards, A., and Atkinson, J.P. 2004. Mutations in CD46, a complement regulatory protein, predispose to atypical HUS. Trends Mol. Med. 10:226-231.

12. Richards, A., et al. 2003. Mutations in human complement regulator, membrane cofactor protein (CD46), predispose to development of familial hemolytic uremic syndrome. Proc. Natl. Acad. Sci. U. S. A. 100:12966-12971.

13. Holt, D.S., et al. 2001. Targeted deletion of the CD59 gene causes spontaneous intravascular hemolysis and hemoglobinuria. Blood. 98:442-449.

14. Pratt, J.R., Basheer, S.A., and Sacks, S.H. 2002. Local synthesis of complement component $\mathrm{C} 3$ regulates acute renal transplant rejection. Nat. Med. 8:582-587. 
15. Thurman, J., Lucia, M., Ljubanovic, D., and Holers, V. 2005. Acute tubular necrosis is characterized by activation of the alternative pathway of complement. Kidney Int. 67:524-530.

16. Li, B., et al. 1993. Mouse Crry/p65. Characterization of monoclonal antibodies and the tissue distribution of a functional homologue of human MCP and DAF. J. Immunol. 151:4295-4305.

17. Lin, F., et al. 2001. Tissue distribution of products of the mouse decay-accelerating factor (DAF) genes. Exploitation of a Daf1 knock-out mouse and site-specific monoclonal antibodies. Immunology. 104:215-225.

18. Qin, X., et al. 2001. Genomic structure, functional comparison, and tissue distribution of mouse Cd59a and Cd59b. Mamm. Genome. 12:582-589.

19. Yamada, K., Miwa, T., Liu, J., Nangaku, M., and Song, W.C. 2004. Critical protection from renal ischemia reperfusion injury by CD55 and CD59. J. Immunol. 172:3869-3875.

20. Turnberg, D., et al. 2004. CD59a deficiency exacerbates ischemia-reperfusion injury in mice. Am.J. Pathol. 165:825-832.

21. Schiller, B., et al. 2001. Expression of a soluble complement inhibitor protects transgenic mice from antibody-induced acute renal failure. J. Am. Soc. Nephrol. 12:71-79.

22. Farrar, C.A., Wang, Y., Sacks, S.H., and Zhou, W. 2004. Independent pathways of P-selectin and complement-mediated renal ischemia/reperfusion injury. Am. J. Pathol. 164:133-141.

23. Quigg, R.J., et al. 1995. Complement regulation in the rat glomerulus: Crry and CD59 regulate complement in glomerular mesangial and endothelial cells. Kidney Int. 48:412-421.

24. Ashworth, S.L., Sandoval, R.M., Hosford, M., Bamburg, J.R., and Molitoris, B.A. 2001. Ischemic injury induces ADF relocalization to the apical domain of rat proximal tubule cells. Am. J. Physiol. Renal Physiol. 280:F886-F894.

25. Molitoris, B.A., Geerdes, A., and McIntosh, J.R. 1991. Dissociation and redistribution of $\mathrm{Na}^{+}, \mathrm{K}^{+}$ ATPase from its surface membrane actin cytoskeletal complex during cellular ATP depletion. J. Clin. Invest. 88:462-469.

26. Woroniecki, R., Ferdinand, J.R., Morrow, J.S., and Devarajan, P. 2003. Dissociation of spectrinankyrin complex as a basis for loss of Na-K-ATPase polarity after ischemia. Am. J. Physiol. Renal Physiol. 284:F358-F364.

27. Dustin, M.L. 2002. Shmoos, rafts, and uropods- the many facets of cell polarity. Cell. 110:13-18.

28. Nomura, A., et al. 1995. Tubulointerstitial injury induced in rats by a monoclonal antibody that inhibits function of a membrane inhibitor of complement. J. Clin. Invest. 96:2348-2356.

29. Ren, G., Doshi, M., Hack, B.K., Alexander, J.J., and Quigg, R.J. 2003. Rat glomerular epithelial cells produce and bear factor $\mathrm{H}$ on their surface that is up-regulated under complement attack. Kidney Int. 64:914-922.

30. Passwell, J., Schreiner, G.F., Nonaka, M., Beuscher, H.U., and Colten, H.R. 1988. Local extrahepatic expression of complement genes C3, factor B, C2, and $\mathrm{C} 4$ is increased in murine lupus nephritis. J. Clin. Invest. 82:1676-1684.

31. Takada, M., Nadeau, K.C., Shaw, G.D., Marquette, K.A., and Tilney, N.L. 1997. The cytokine-adhesion molecule cascade in ischemia/reperfusion injury of the rat kidney. Inhibition by a soluble P-selectin ligand. J. Clin. Invest. 99:2682-2690.

32. Park, P., et al. 2001. Inhibiting the complement system does not reduce injury in renal ischemia reperfusion. J. Am. Soc. Nephrol. 12:1383-1390.

33. Girardi, G., Redecha, P., and Salmon, J.E. 2004. Heparin prevents antiphospholipid antibodyinduced fetal loss by inhibiting complement activation. Nat. Med. 10:1222-1226.

34. Baker, P.J., Adler, S., Yang, Y., and Couser, W.G. 1984. Complement activation by heat-killed human kidney cells: formation, activity, and stabilization of cellbound C3 convertases. J. Immunol. 133:877-881.

35. David, S., et al. 1997. Alternative pathway complement activation induces proinflammatory activity in human proximal tubular epithelial cells. Nephrol. Dial. Transplant. 12:51-56.

36. Molitoris, B.A., Chan, L.K., Shapiro, J.I., Conger, J.D., and Falk, S.A. 1989. Loss of epithelial polarity: a novel hypothesis for reduced proximal tubule $\mathrm{Na}+$ transport following ischemic injury. J. Membr. Biol. 107:119-127.

37. Sheridan, A.M., and Bonventre, J.V. 2000. Cell biology and molecular mechanisms of injury in ischemic acute renal failure. Curr. Opin. Nephrol. Hypertens. 9:427-434.

38. Ichida, S., Yuzawa, Y., Okada, H., Yoshioka, K., and Matsuo, S. 1994. Localization of the complement regulatory proteins in the normal human kidney. Kidney Int. 46:89-96.

39. Kwon, O., et al. 1999. Sodium reabsorption and distribution of $\mathrm{Na}+/ \mathrm{K}^{+-A T P a s e}$ during postischemic injury to the renal allograft. Kidney Int. 55:963-975.

40. Stahel, P.F., Morganti-Kossmann, M.C., and Kossmann, T. 1998. The role of the complement system in traumatic brain injury. Brain Res. Brain Res. Rev. 27:243-256.

41. Taube, C., et al. 2003. Inhibition of complement activation decreases airway inflammation and hyperresponsiveness. Am. J. Respir. Crit. Care Med. 168:1333-1341.

42. Nataf, S., Carroll, S.L., Wetsel, R.A., Szalai, A.J., and Barnum, S.R. 2000. Attenuation of experimental autoimmune demyelination in complement-deficient mice. J. Immunol. 165:5867-5873.

43. Vakeva, A., et al. 1994. Time course of complement activation and inhibitor expression after ischemic injury of rat myocardium. Am. J. Pathol. 144:1357-1368.

44. Vakeva, A., and Meri, S. 1998. Complement activation and regulator expression after anoxic injury of human endothelial cells. APMIS. 106:1149-1156.

45. Xu, C., et al. 2000. A critical role for murine complement regulator crry in fetomaternal tolerance. Science. 287:498-501.

46. Nadasdy, T., Laszik, Z., Blick, K.E., Johnson, D.L., and Silva, F.G. 1994. Tubular atrophy in the endstage kidney: a lectin and immunohistochemical study. Hum. Pathol. 25:22-28.

47. Ohta, R., et al. 2004. Mouse complement receptor-related gene y/p65-neutralized tumor vaccine induces antitumor activity in vivo. J. Immunol. 173:205-213

48. Thurman, J.M., et al. 2005. A novel inhibitor of the alternative complement pathway prevents antiphospholipid antibody-induced pregnancy loss in mice. Mol. Immunol. 42:87-97.

49. Sorribas, V., et al. 2001. Gentamicin causes endocytosis of $\mathrm{Na} / \mathrm{Pi}$ cotransporter protein (NaPi-2). Kidney Int. 59:1024-1036.

50. Molitoris, B.A., Dahl, R., and Geerdes, A. 1992. Cytoskeleton disruption and apical redistribution of proximal tubule $\mathrm{Na}(+)-\mathrm{K}(+)$-ATPase during ischemia. Am. J. Physiol. 263:F488-F495.

51. Levi, M., Molitoris, B.A., Burke, T.J., Schrier, R.W., and Simon, F.R. 1987. Effects of vitamin D-induced chronic hypercalcemia on rat renal cortical plasma membranes and mitochondria. Am. J. Physiol. 252:F267-F275.

52. Molitoris, B.A., and Simon, F.R. 1985. Renal cortical brush-border and basolateral membranes: cholesterol and phospholipid composition and relative turnover. J. Membr. Biol. 83:207-215.

53. Aufricht, C., et al. 2002. Ischemic conditioning prevents $\mathrm{Na}, \mathrm{K}$-ATPase dissociation from the cytoskeletal cellular fraction after repeat renal ischemia in rats. Pediatr. Res. 51:722-727.

54. Sinha, D., Wang, Z., Price, V.R., Schwartz, J.H., and Lieberthal, W. 2003. Chemical anoxia of tubular cells induces activation of c-Src and its translocation to the zonula adherens. Am. J. Physiol. Renal Physiol. 284:F488-F497. 